\title{
Cross-correlation of the Two Micron Galactic Survey with IRAS
}

\author{
T. Mahoney, F. Garzón, X. Calbet, P. Hammersley, M. J. Selby
}

Instituto de Astrofísica de Canarias, E-38200 La Laguna, Tenerife (Spain)

The Two Micron Galactic Survey (TMGS) is described by Calbet et al. (this symposium) and Mahoney et al. (1990). It has been crosscorrelated with the IRAS point source catalogue (IRPS) using a 15arcsec search radius, and preliminary results are presented here.

There were many one-to-one correspondences, but source confusion was severe close to the plane. At the limiting magnitude of the TMGS $\left(m_{K}=+10.5^{\mathrm{m}}\right)$ and with a galactocentric distance of $8 \mathrm{kpc}$ and extinction of $0.0002 \mathrm{mag} \mathrm{pc}^{-1}$ a typical M3 III giant with $M_{K}=-5.5^{\mathrm{m}}$ will have an apparent magnitude of $m_{K}=+10.6^{\mathrm{m}}$. So the TMGS should see such objects almost as far as the Galactic centre. The IRPS sources examined correspond to three regions scanned by the TMGS. These zones are roughly 1 degree wide in declination and 2-3 hours long in right ascension. They are centred at $\delta=-1^{\circ},-22^{\circ}$ and $-30^{\circ}$ (approximately) and are centred in RA on the Galactic plane.

Colour-colour plots and histograms of source distributions of the IRPS data alone for each of the three regions were compared with similar plots and histograms for sources correlated with the TMGS. For IRAS-only data, the Galactic plane is seen at $\delta=-1^{\circ}$. The bulge is evident at $l=-22^{\circ}$ and dominates at the Galactic centre. In 12-25-65 $\mu \mathrm{m}$ diagrams all sources above the $F_{\nu}(12 \mu \mathrm{m})=F_{\nu}(25 \mu \mathrm{m})$ line are stellar, whereas galaxies and "Galactic components" lie to the left of the $F_{\nu}(25 \mu \mathrm{m})=F_{\nu}(60 \mu \mathrm{m})$ line. Stellar sources are of three types: photospheres, with $F_{\nu}(12 \mu \mathrm{m})>3 F_{\nu}(25 \mu \mathrm{m})$, circumstellar dust shells (CDS), with $F_{\nu}(25 \mu \mathrm{m})<F_{\nu}(12 \mu \mathrm{m})<3 F_{\nu}(25 \mu \mathrm{m})$, and "disk and bulge stars", which extend the stellar region of the colour-colour diagram below the $F_{\nu}(12 \mu \mathrm{m})=F_{\nu}(25 \mu \mathrm{m})$ line. Nearly all source types are represented where all the sources in the zones are included. Closer in towards the Galactic plane, however, only the redder CDS's remain, along with the Galactic components. Photospheres become much scarcer.

For cross-correlation we used high-quality IRAS sources for $|b|<$ $15^{\circ}$ and applied two criteria: (i) $6.0 \mathrm{Jy}<F_{\nu}(12 \mu \mathrm{m})<0.6 \mathrm{Jy}$, and (ii) the redness parameter $R=F_{\nu}(25 \mu \mathrm{m}) / F_{\nu}(12 \mu \mathrm{m})>0.7$ (Habing 1986). 
The plane is still evident at $\delta=-1^{\circ}$, but the bulge is clearly seen at $\delta=-22^{\circ}$. Source confusion, particularly in the IRAS data, is the principal reason for the poor showing of the bulge in the $\delta=-30^{\circ}$ zone. It seems, nevertheless, that the TMGS is seeing into the bulge. New 12-25-60 $\mu \mathrm{m} \mathrm{c-c}$ plots were made for the remaining sources. CDS's cluster about the -0.1 abscissa value, although this is not so evident for the $-30^{\circ}$ zone, due, probably, to confusion problems. In common with Habing, we find with cross-correlation that the plane and bulge become more pronounced as the two criteria are applied, hence confirming that the TMGS is detecting bulge sources.

\section{References}

Habing, H 1986, in F. P. Israel (ed.), Light on Dark Matter. Proceedings of the First IRAS Conference, Noordwijk, The Netherlands. Dordrecht: Reidel.

Mahoney, T., Selby, M. J., Garzón, F., Hammersley, P. and Calbet, X. 1990, in B. J. Jarvis and D. M. Terndrup (eds.), Bulges of galaxies. ESO/CTIO workshop, La Serena, Chile. 